\title{
FMBEM 求解直圆柱线性波绕射问题
}

\author{
林志良* \\ 上海交通大学船舶海洋与建筑工程学院海洋工程国家重点试验室, 上海 200240 \\ *E-mail: linzhiliang@sjtu.edu.cn \\ 收稿日期: 2010-11-08; 接受日期: 2010-12-16 \\ 国家自然科学基金资助项目(批准号: 50739004, 50509016)
}

\begin{abstract}
摘要运用快速多极子边界元法(Fast Multipole Boundary Element Method, FMBEM)求得单圆柱在线性波浪 中的绕射问题的数值解. 所谓的快速多极子边界元法就是采用快速多极子法(Fast Multipole Method, FMM)加 速传统边界元法的求解速度. 在文中通过求解二维的 Helmholtz 方程证明 FMBEM 法具有高精度和高效率, 适 用于求解大规模的数值问题. 另外, 给出了单圆柱线性平面波绕射问题中相关水动力学系数的数值计算结果.
\end{abstract}

关键词快速多极子边界元法, 波浪绕射, Helmholtz 方程

PACS: $\quad$ 47.35.Lf, 71.15.Ap, 92.10.Hm, 92.10.Lq

在海洋工程中，波浪与垂直柱子相互作用已有 深入的研究. 1954 年, Maccamy 和 Fuchs ${ }^{[1]}$ 给出了在线 性波浪中单个垂直圆柱绕射问题的解析解. 从那以 后, 有许多圆柱平面波绕射问题相关的数值计算报

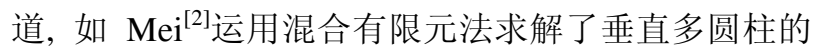
平面波绕射问题, 而 $\mathrm{Au}$ 和 Brebbia ${ }^{[3]}$ 运用传统的边界 元法(Boundary Element Method, BEM)求解了同类问 题. 边界元法只需离散所求解问题的边界, 从而使问 题的计算维数减少了一维, 并且具有计算精度高的 优点. 然而, 运用传统的边界元法需要花费大量的 $\mathrm{CPU}$ 时间生成一个 $N \times N$ 阶的满阵 $(N$ 为边界离散节 点的个数), 同时需要占用大量的内存空间储存这个 系数矩阵. 求解该满阵线性方程组同样需要耗费大 量的计算时间和内存空间, 采用直接法求解需要 $O\left(N^{3}\right)$ 的计算量级和 $O\left(N^{2}\right)$ 的存储量级, 而采用迭 代法求解的计算量级为 $O\left(N^{2}\right)$. 所以当求解大规模 问题时, 传统边界元法的计算效率已经成为一个严
重的缺陷. 而最近可采用一种叫作快速多极子法 (Fast Multipole Method, FMM)的方法来加速传统边 界元法的矩阵计算, 可有效降低内存消耗, 克服传统 边界元法的局限性.

快速多极子法最初由 Rokhlin ${ }^{[4]}$ 于 1985 年提出的 一种求解二维 Laplace 方程的快速算法, 并已被誉为 20 世纪十大快速算法之一[5]. 快速多极子法和传统 边界元法相结合产生快速多极子边界元法(FMBEM). 当边界上节点数 $N$ 大于数百或上千时, 该新方法的 计算速度要明显快于传统的边界元法. 在 Liu 和 Nishimura $^{[6]}$ 中, 已对 FMBEM 及其主要求解过程进行 了详细的介绍, 而关于 FMBEM 应用的全面综述可参 见 Nishimura ${ }^{[7]}$.

本文应用 FMBEM 求解单圆柱线性平面波绕射 问题, 并将计算结果与传统边界元法的计算结果相 比较, 验证 FMBEM 的高计算效率; 也将数值计算结 果同 $\mathrm{Mei}^{[8]}$ 中所提供的解析解相比较, 验证新方法的

引用格式: 林志良. FMBEM 求解直圆柱线性波绕射问题. 中国科学: 物理学 力学 天文学, 2011, 41: 155-160 Lin Z L. Diffraction of linear waves around a vertical circular cylinder using the FMBEM (in Chinese). Sci Sin Phys Mech Astron, 2011, 41: 155-160, doi: 10.1360/132010-1067 
计算精度. 同时文中给出了单圆柱线性平面波绕射 问题中相关水动力学系数的数值计算结果.

\section{1 问题描述}

如图 1 所示, 在 $z$ 轴方向上有一个固定圆柱从等 深的水底垂直穿出水面, 而在 $x y$ 平面上有个单频入 射长峰波, 而坐标原点定义在圆柱体的中轴线与平 均水面交接处. 假设流体无粘不可压, 并且无旋, 满 足势流理论. 因此, 我们可以引入速度势并定义总速 度势为 $\Phi$, 入射波速度势为 $\Phi_{I}$ 以及波浪散射势为 $\Phi_{S}$. 入射波的波数表示为 $k$, 且沿 $x$ 轴方向和 $y$ 轴方 向的分量分别为 $k_{x}$ 和 $k_{y}$, 入射波频率为 $\omega$, 水深为 $h$, 波幅为 $A$, 圆柱半径为 $a$, 时间为 $t$, 而重力加速度 为 $g$.

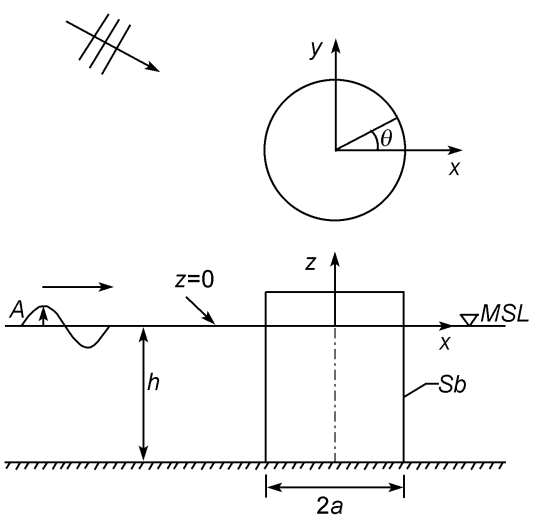

图 1 坐标系统示意图

在线性波理论中, 总速度势 $\Phi$ 在整个流场 $(\Omega)$ 中 应满足 Laplace 方程, 即

$$
\nabla^{2} \Phi=0,
$$

并在平均水面 $(z=0)$ 上满足线性化自由面条件

$$
\frac{\partial^{2} \Phi}{\partial t^{2}}+g \frac{\partial \Phi}{\partial z}=0,
$$

而在水底 $(z=-h)$ 和圆柱表面 $\left(S_{b}\right)$ 上满足不可穿透条 件, 分别为

$$
\frac{\partial \Phi}{\partial z}=0
$$

和

$$
\frac{\partial \Phi}{\partial n}=0,
$$

其中 $n$ 表示圆柱表面的单位法向量

对于线性单频波问题，速度势可做如下分解

$$
\begin{aligned}
\Phi(x, y, z, t) & =\phi(x, y) Z(z) \mathrm{e}^{-\mathrm{i} \omega t}, \\
\Phi_{I}(x, y, z, t) & =\phi^{I}(x, y) Z(z) \mathrm{e}^{-\mathrm{i} \omega t}, \\
\Phi_{S}(x, y, z, t) & =\phi^{S}(x, y) Z(z) \mathrm{e}^{-\mathrm{i} \omega t},
\end{aligned}
$$

其中

$$
Z(z)=\frac{\cosh k(z+h)}{\cosh k h} .
$$

在这个简化过程中, 水底边界条件自动满足, 而线性 自由面边界条件满足色散关系

$$
\omega^{2}=g k \tanh k h .
$$

因此，原问题可以简化为只在自由水面上的二 维问题. 而且入射波速度势 $\Phi_{I}$ 给定, 从而未知量 $\phi^{S}(x, y)$ 的控制方程为 Helmholtz 方程

$$
\nabla^{2} \phi^{S}+k^{2} \phi^{S}=0,
$$

并且在圆柱表面满足边界条件

$$
\frac{\partial \phi^{S}}{\partial n}=-\frac{\partial \phi^{I}}{\partial n} \quad \text { 在 } r=a \text { 上, }
$$

无穷远处满足所谓的 Sommerfeld 条件

$$
\lim _{k r \rightarrow \infty}(k r)^{1 / 2}\left(\frac{\partial \phi^{S}}{\partial r}-i k \phi^{S}\right)=0,
$$

其中 $r$ 是径向坐标，而 $\mathrm{i}=\sqrt{-1}$.

给定的线性平面入射波的速度势表示为

$$
\Phi_{I}=-\frac{\mathrm{i} g A}{\omega} Z(z) \mathrm{e}^{\mathrm{i}\left(k_{x} x+k_{y} y-\omega t\right)} .
$$

而总速度势与散射波速度势和入射波速度势的关系 如下:

$$
\Phi=\Phi_{I}+\Phi_{S}, \quad \phi=\phi^{I}+\phi^{S} .
$$

因此，垂直圆柱单频线性波绕射问题由控制方程(10) 和边界条件(11), (12)组成. 通过求解这个边值问题, 我们可以求得未知量 $\phi^{S}$ 和 $\Phi$. 从而流场中流体的速 度、自由表面的升高以及动压力可以通过

$$
\begin{gathered}
v=\nabla \Phi, \\
\eta=\frac{\mathrm{i} \omega}{g} \phi, \\
p=-\rho \frac{\partial \Phi}{\partial t},
\end{gathered}
$$

分别计算得到, 其中 $\rho$ 表示流体的密度.

对于平面入射波, 我们总能重新定义一个新坐 
标使得波沿着 $x$ 轴的正方向传播, 也就说可以令 $k_{x}=k$ 和 $k_{y}=0$. 因此，假设平面入射波沿着 $x$ 轴的 正方向传播. 对于一个单位的高度水平切片, 在波浪 传播方向上的所受的力为

$$
\frac{\mathrm{d} F_{x}}{\mathrm{~d} z}=-a \int_{0}^{2 \pi} p(a, \theta, z) \cos (\theta) \mathrm{d} \theta,
$$

其中 $p(a, \theta, z)$ 表示圆柱表面上的压力. 作用在圆柱 上无量纲力可定义为

$$
R(k, a)=\frac{\frac{\mathrm{d} F_{x}}{\mathrm{~d} z}}{2 \pi a \rho g A Z(z) \mathrm{e}^{-\mathrm{i} \omega t}} .
$$

因此, 作用在圆柱上沿水平方向的总力为

$$
F_{x}=\int_{-h}^{0} \frac{\mathrm{d} F_{x}}{\mathrm{~d} z} \mathrm{~d} z=2 \pi a R(k, a) \rho g A \mathrm{e}^{-\mathrm{i} \omega t} \tanh (k h) / k \text {. }
$$

根据 $\mathrm{Mei}^{[8]}$, 每一单位高度柱体的总受力可以表 示为

$$
\operatorname{Re}\left(\frac{\mathrm{d} F_{x}}{\mathrm{~d} z}\right)=\rho \pi a^{2}\left(C_{\mathrm{M}} \dot{U}+\omega C_{\mathrm{D}} U\right),
$$

其中 $C_{\mathrm{M}}$ 为惯性系数, $C_{\mathrm{D}}$ 为阻力系数, 而 $U$ 是不存 在圆柱体时, $x=0$ 处的入射波速度.

根据(13), (15), (18), (19)和(21), 我们可以得到

$$
C_{\mathrm{M}}=-\frac{2 R_{\mathrm{i}}}{k a}, \quad C_{\mathrm{D}}=\frac{2 R_{\mathrm{r}}}{k a},
$$

其中 $R_{\mathrm{r}}$ 和 $R_{\mathrm{i}}$ 分别表示无量纲受力 $R(k, a)$ 的实部和虚 部.

根据 $\mathrm{Mei}^{[8]}$, 我们还可以得到 $\frac{\mathrm{d} F_{x}}{\mathrm{~d} z}, C_{\mathrm{M}}$ 和 $C_{\mathrm{D}}$ 的 精确解表达式, 即

$$
\begin{aligned}
\frac{\mathrm{d} F_{x}}{\mathrm{~d} z} & =\frac{4 A}{k a} \frac{\rho g a}{H_{1}^{\prime}(k a)} Z(z) \mathrm{e}^{-\mathrm{i} \omega t}, \\
C_{\mathrm{M}} & =\frac{4}{\pi} \frac{Y_{1}^{\prime}(k a)}{(k a)^{2}\left|H_{1}^{\prime}(k a)\right|^{2}}, \\
C_{\mathrm{D}} & =\frac{4}{\pi} \frac{J_{1}^{\prime}(k a)}{(k a)^{2}\left|H_{1}^{\prime}(k a)\right|^{2}},
\end{aligned}
$$

其中 $H_{1}^{\prime}(s) \equiv \mathrm{d} H_{1}^{(1)} / \mathrm{d} s, \quad J_{1}^{\prime}(s) \equiv \mathrm{d} J_{1} / \mathrm{d} s$ 以及 $Y_{1}^{\prime}(s) \equiv \mathrm{d} Y_{1} /$ $\mathrm{d} s$, 而 $H_{1}^{(1)}$ 为第一类一阶 Hankel 函数, $J_{1}$ 和 $Y_{1}$ 分别 为第一类和第二类一阶 Bessel 函数. 根据(19), 无量 纲受力 $R(k, a)$ 的精确解为

$$
R(k, a)=\frac{2}{\pi k a H_{1}^{\prime}(k a)} .
$$

因此, 我们可以将数值计算结果与精确解相比较, 从 而验证计算精度.

\section{FMBEM 求解二维 Helmholtz 方程}

在线性波理论假设下，圆柱波浪绕射问题可简 化为一个关于散射波速度势 $\phi^{s}$ 的二维 Helmholtz 方 程边值问题, 由控制方程(10)和边界条件(11), (12)组 成. 从而可以运用快速多极子边界元法进行求解, 为 了简便, 将控制方程和边界条件重写为

$$
\left(\nabla^{2}+k^{2}\right) \phi(\boldsymbol{x})=0, \quad \forall \boldsymbol{x} \in \Omega,
$$

满足边界条件

$$
q(\boldsymbol{x}) \equiv \frac{\partial \phi}{\partial n}(\boldsymbol{x})=\bar{q}(\boldsymbol{x}), \quad \forall \boldsymbol{x} \in S,
$$

其中 $\boldsymbol{x}$ 表示流体计算域内的坐标为 $(x, y)$ 的点, 而在边 界条件中, 上划线表示参量给定. 通过选择方程(27) 的基本解, 可以使得无穷远处的边界条件自动满足.

对于二维的 Helmholtz 方程边值问题, 可以直接 得到边界积分方程

$$
\begin{gathered}
\phi(\boldsymbol{x})=\int_{\Gamma}(G(\boldsymbol{x}-\boldsymbol{y}) q(\boldsymbol{y})-F(\boldsymbol{x}-\boldsymbol{y}) \phi(\boldsymbol{y})) \mathrm{d} \Gamma(\boldsymbol{y}), \\
\forall \boldsymbol{x} \in \Omega,
\end{gathered}
$$

其中 $G$ 是二维 Helmholtz 方程的基本解，表示为

$$
G(\boldsymbol{x}-\boldsymbol{y})=\frac{\mathrm{i}}{4} H_{0}^{(1)}(k|\boldsymbol{x}-\boldsymbol{y}|),
$$

而相应的有

$$
F(\boldsymbol{x}-\boldsymbol{y})=\frac{\partial G(\boldsymbol{x}-\boldsymbol{y})}{\partial n} .
$$

在方程(30)式中, $\boldsymbol{x}$ 表示从坐标原点 $O$ 指向配置点 $\boldsymbol{x}$ 的矢 量, $H_{0}^{(1)}$ 表示第一类零阶 Hankel 函数, 而 $\boldsymbol{y}$ 表示场点.

\subsection{FMBEM 基本公式}

快速多极子边界元法中, 有若干个重要的公式 推导, 包括多极子动量矩(Multipole Moment)、动量矩 转移(Moment-to-Moment Translation, M2M)、局部展 开系数(Local Expansion)、动量矩到局部展开系数的 转移(Moment-to-Local Translation, M2L)和局部展开 系数的转移(Local-to-Local Translation, L2L), 详细内 容请参见 Liu 和 Nishimura ${ }^{[6]}$. 在这里, 仅简要给出二 维 Helmholtz 方程相应的公式, 而公式中所用到的相 关点的位置如图 2 所示. 


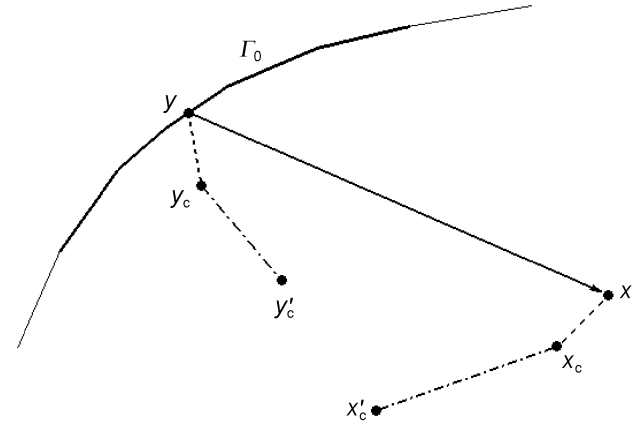

图 2 FMBEM 中的几个相关点

根据 Graf 叠加原理 ${ }^{[9]}$, 可以得到

$$
G(\boldsymbol{x}-\boldsymbol{y})=\frac{\mathrm{i}}{4} \sum_{n=-\infty}^{\infty} O^{n}\left(\boldsymbol{y}_{c} \boldsymbol{x}\right) I^{-n}\left(\boldsymbol{y}_{c} \boldsymbol{y}\right),
$$

其中, 函数 $O^{n}$ 和 $I^{n}$ 定义为

$$
\begin{aligned}
& O^{n}\left(\boldsymbol{y}_{c} \boldsymbol{x}\right)=i^{n} H_{n}^{(1)}(k r) \mathrm{e}^{\mathrm{i} n \theta}, \\
& I^{n}\left(\boldsymbol{y}_{c} \boldsymbol{x}\right)=(-i)^{n} J_{n}(k r) \mathrm{e}^{\mathrm{i} n \theta} .
\end{aligned}
$$

在表达式(33)中, $J_{n}$ 表示 Bessel 函数, $(r, \theta)$ 表示矢 量 $\boldsymbol{y}_{c} \boldsymbol{x}$ 的极坐标.

对于核函数为 $G$ 的边界积分表达式有

$$
\int_{\Gamma_{0}} G(\boldsymbol{x}-\boldsymbol{y}) q(\boldsymbol{y}) \mathrm{d} \Gamma=\frac{\mathrm{i}}{4} \sum_{n=-\infty}^{\infty} O^{n}\left(\boldsymbol{y}_{c} \boldsymbol{x}\right) M_{-n}\left(\boldsymbol{y}_{c}\right),
$$

其中多极子动量矩为

$$
M_{n}\left(\boldsymbol{y}_{c}\right)=\int_{\Gamma_{0}} I^{n}\left(\boldsymbol{y}_{c} \boldsymbol{y}\right) q(\boldsymbol{y}) \mathrm{d} \Gamma .
$$

经过推导, 可以得到多极子动量矩转移 (M2M) 表达式

$$
M_{n}\left(\boldsymbol{y}_{c}^{\prime}\right)=\sum_{v=-\infty}^{\infty} I^{n-v}\left(\overrightarrow{\boldsymbol{y}_{c}^{\prime} \boldsymbol{y}_{c}}\right) M_{v}\left(\boldsymbol{y}_{c}\right) .
$$

边界积分(34)的局部展开(Local Expansion)表达式

$$
\int_{\Gamma_{0}} G(\boldsymbol{x}-\boldsymbol{y}) q(\boldsymbol{y}) \mathrm{d} \Gamma=\frac{\mathrm{i}}{4} \sum_{n=-\infty}^{\infty}(-1)^{n} I^{n}\left(\boldsymbol{x}_{c} \boldsymbol{x}\right) L_{-n}\left(\boldsymbol{x}_{c}\right) .
$$

动量矩到局部展开系数的转移 $(\mathrm{M} 2 \mathrm{~L})$ 表达式

$$
L_{n}\left(\boldsymbol{x}_{c}\right)=\sum_{v=-\infty}^{\infty} O^{n-v}\left(\boldsymbol{y}_{c}^{\prime} \boldsymbol{x}_{c}\right) M_{v}\left(\boldsymbol{y}_{c}^{\prime}\right),
$$

以及局部展开系数转移(L2L)表达式

$$
L_{n}\left(\boldsymbol{x}_{c}^{\prime}\right)=\sum_{v=-\infty}^{\infty} I^{n-v}\left(\overrightarrow{\boldsymbol{x}_{c}^{\prime} \boldsymbol{x}_{c}}\right) L_{v}\left(\boldsymbol{x}_{c}\right) .
$$

对于核函数为 $F$ 的边界积分表达式有

$$
\int_{\Gamma_{0}} F(\boldsymbol{x}-\boldsymbol{y}) \phi(\boldsymbol{y}) \mathrm{d} \Gamma=\frac{\mathrm{i}}{4} \sum_{n=-\infty}^{\infty} O^{n}\left(\boldsymbol{y}_{c} \boldsymbol{x}\right) N_{-n}\left(\boldsymbol{y}_{c}\right),
$$

其中多极子动量矩定义为

$$
N_{n}\left(\boldsymbol{y}_{c}\right)=\int_{\Gamma_{0}} \frac{\partial I^{n}\left(\boldsymbol{y}_{c} \boldsymbol{y}\right)}{\partial n} \phi(\boldsymbol{y}) \mathrm{d} \Gamma .
$$

而对于其他表达式与核函数为 $G$ 的表达式一样, 只需用 $N_{n}$ 替代 $M_{n}$.

\subsection{FMBEM 求解步骤}

FMBEM 的主要思想是将传统边界元法中离散 节点与节点之间的相互作用转换成单元块与单元块 间的相互作用, 从而大大地加快求解速度. 在这里我 们简要介绍 FMBEM 主要的求解步骤, 详细介绍同样 请参见 Liu 和 Nishimura ${ }^{[6]}$.

第一步: 与传统边界元法一样, 对边界 $\Gamma$ 进行单 元离散.

第二步: 构建离散单元的树状结构, 确定叶子单 元、单元块间的父子关系以及不同层级单元块之间的 远近关系.

第三步：向上传递过程，计算每个单元块的多极 动量矩, 这个过程从最细层级开始并逐层向上聚合.

第四步：向下传递过程，从树状结构的第 2 层级 开始逐层向下计算每个单元块的局部展开系数, 最 终得到位于每个叶子单元中心的局部展开系数.

第五步：将近场和远场的贡献相加，配置点相应 的近场节点的作用采用传统边界元法直接计算, 而 远场节点的作用则通过配置点所在叶子单元中心的 局部展开系数计算得到.

第六步: 运用广义极小残值迭代算法(Generalized Minimum Residual Method, GMRES $)^{[10]}$ 计算系数矩阵 方程中的未知列向量. 在 GMRES 算法, 仅用近场离 散节点之间的影响系数组成一个新的稀疏系数矩阵, 而右端项是通过第三步至第五步快速计算得到. 如 果迭代算法的误差小于设定的误差值，当前的列向 量为所求的值; 如果不满足误差要求, 则更新待求列 向量，并回到第三步继续计算.

\section{3 数值计算结果}

\subsection{FMBEM 与传统的 BEM 的比较}

令波数 $k=k_{x}=1.0$, 圆柱的半径 $a=1.0$, 同时用 传统的边界元法和快速多极子边界元法求解, 采用 常数单元离散边界. 在 FMBEM 中, 所有的快速多极 子方法的基本公式中, 需要设置一个截断数 $P$ 来代 替无穷项相加, 在这里令 $P=10$; 在构建树状结构时, 
设置每个叶子单元所包含的最多节点数为 20 个; 而 GMRES 迭代算法中的误差判断标准设置为 $10^{-8}$. 在 本文中, 所有的 Fortran 程序计算都是在一台 $3.0 \mathrm{GHz}$ CPU, 2. 0GB RAM 的台式计算机完成的.

在表 1 中, 分别给出运用 FMBEM 和传统的 BEM 求解得到的惯性系数 $C_{\mathrm{M}}$, 阻力系数 $C_{\mathrm{D}}$ 和无量纲受力 $|2 \pi a R|$ 的值. 与相应的解析解比较, 可知两种方法得 到的数值计算结果随着边界离散单元数目 $N$ 的增加 是收玫于精确解的. 表 2 给出了两种方法在不同离散 单元情况下所需的计算时间, 并在图 3 中给出了计算 时间关于离散单元数目的变化曲线. 当 $N$ 少于 200 时, 传统 BEM 所需要的计算时间少于 FMBEM. 但是，随 着 $N$ 的增加，传统边界元法所需的计算时间成几何 增长, 而采用 FMBEM, 计算时间却几乎是线性增长. 这说明了快速多极子边界元法将边界元法的计算时 间降低到 $O(N)$ 量级, 成功地克服了传统边界元方法 计算时间长、计算效率低下的缺点. 在台式机上，当 离散单元数达到 9600 左右, 也就是不到一万个离散 节点, 传统边界元法早已失效, 而运用快速多极子边 界元法进行求解, 所需的时间仅仅需要 $43.7 \mathrm{~s}$, 少于 $1 \mathrm{~min}$. 这很好地证明了快速多极边界元法求解大规 模数值问题时所具有的高效性.

\section{2 波浪绕射的数值结果}

对于不同的圆柱半径 $a$ 和波数 $k$, 表 3 分别给出 了惯性系数 $C_{\mathrm{M}}$, 阻力系数 $C_{\mathrm{D}}$ 和无量纲受力 $|2 \pi a R|$ 的 值. 从表中可知, $C_{\mathrm{M}}, C_{\mathrm{D}}$ 和 $|R|$ 是 $k a$ 的函数. 图 4 给出

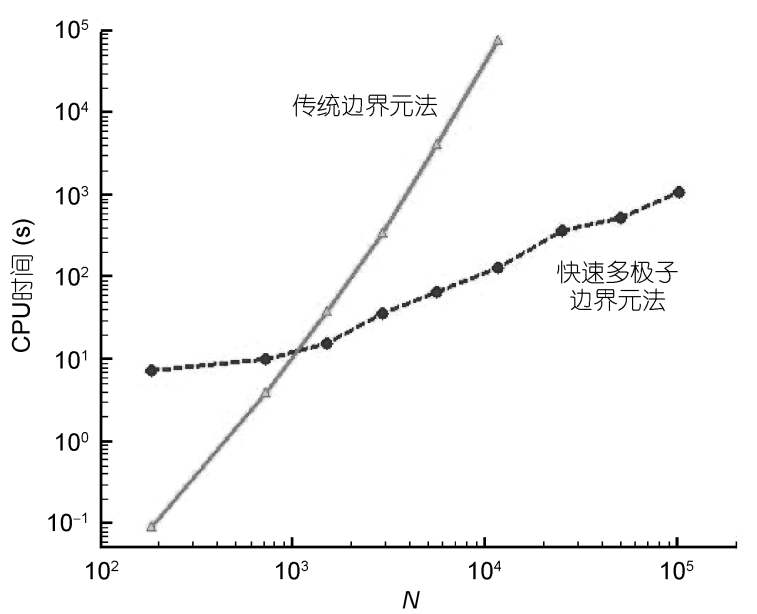

图 3 CPU 计算时间比较

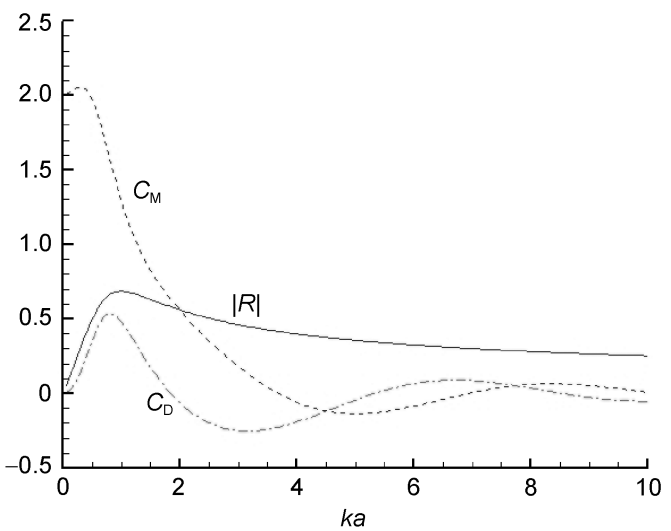

图 4 惯性系数、阻力系数和无量纲力随着 $k a$ 的变化曲线

了惯性系数 $C_{\mathrm{M}}$, 阻力系数 $C_{\mathrm{D}}$ 和无量纲受力 $|2 \pi a R|$ 随 着 $k a$ 的变化曲线.

表 1 在 $k_{x}=k=1.0, a=1.0$ 情况下, $C_{\mathrm{M}}$ 和 $C_{\mathrm{D}}$ 以及 $|2 \pi a R|$ 的比较 ${ }^{\mathrm{a})}$

\begin{tabular}{|c|c|c|c|c|c|c|}
\hline \multirow{2}{*}{$N$} & \multicolumn{2}{|c|}{$C_{\mathrm{M}}$} & \multicolumn{2}{|c|}{$C_{\mathrm{D}}$} & \multicolumn{2}{|c|}{$|2 \pi a R|$} \\
\hline & FMBEM & TBEM & FMBEM & TBEM & FMBEM & TBEM \\
\hline 36 & 1.28319 & 1.28319 & 0.48213 & 0.48213 & 4.3064 & 4.3064 \\
\hline 72 & 1.28428 & 1.28428 & 0.48084 & 0.48084 & 4.3082 & 4.3082 \\
\hline 360 & 1.28469 & 1.28469 & 0.48044 & 0.48044 & 4.30896 & 4.30896 \\
\hline 720 & 1.28471 & 1.28471 & 0.48044 & 0.48044 & 4.30901 & 4.30901 \\
\hline 2400 & 1.28472 & 1.28472 & 0.48044 & 0.48044 & 4.30905 & 4.30905 \\
\hline 7200 & 1.28472 & 1.28472 & 0.48043 & 0.48043 & 4.30905 & 4.30905 \\
\hline 9600 & 1.28472 & & 0.48043 & & 4.30906 & \\
\hline 精确解 & 1.28472 & & 0.48043 & & 4.30906 & \\
\hline
\end{tabular}

a) TBEM: 传统的 BEM

表 $2 \mathrm{CPU}$ 计算时间比较(单位: $\mathrm{s}$ )

\begin{tabular}{cccccccc}
\hline$N$ & 72 & 360 & 1440 & 2400 & 4800 & 9600 \\
\hline FMBEM & 0.2 & 1.3 & 5.6 & 10.7 & 19.9 & 32.6 & 43.8 \\
传统 BEM & 0.05 & 2.4 & 182.0 & 911.7 & 7369.7 & 25642.3 & \\
\hline
\end{tabular}


表 3 在不同的 $\boldsymbol{k}$ 和 $\boldsymbol{a}$ 情况下, $\boldsymbol{C}_{\mathrm{M}}$ 和 $\boldsymbol{C}_{\mathrm{D}}$ 以及 $|\boldsymbol{R}|$ 的比较

\begin{tabular}{ccccc}
\hline$a(\mathrm{~m})$ & $k_{x}\left(\mathrm{~m}^{-1}\right)$ & $C_{\mathrm{M}}$ & $C_{\mathrm{D}}$ & $|R|$ \\
\hline 1.0 & 1.0 & 1.28428 & 0.48084 & 0.68567 \\
1.0 & 3.0 & 0.18055 & -0.24936 & 0.46180 \\
0.5 & 2.0 & 1.28428 & 0.48084 & 0.68567 \\
1.5 & 2.0 & 0.18055 & -0.24936 & 0.46180 \\
\hline
\end{tabular}

\section{4 结论}

本文应用快速多极子法加快传统边界元法计算
二维的 Helmholtz 方程的速度, 成功求解线性平面波 浪中垂直圆柱绕射问题. 通过与传统边界元法的比 较, 快速多极子边界元法具有优越的计算效率, 可以 有效快速求解大规模数值问题, 有效克服传统边界 元法计算效率低下, 计算规模较小的局限性. 而与已 有的解析解比较, FMBEM 可以保证很高的计算精度. FMBEM 具有很高的计算效率和计算精度, 可以快速 求解大规模势流问题, 在海洋工程及其他计算领域 中具有巨大的应用前景.

\title{
参考文献
}

1 MacCamy R C, Fuchs R A. Wave forces on piles: A diffraction theory. U. S. Army Corps of Engineering, Beach Erosion Board, Technical Memorandum, 1954. 69

2 Mei C C. Numerical methods in water-wave diffraction and radiation. Annu Rev Fluid Mech, 1978, 10: 393-416

3 Au M C, Brebbia C A. Diffraction of water waves for vertical cylinders using boundary elements. Appl Math Model, 1983, 7(2): 106-114

4 Rokhlin V. Rapid solution of integral equation of classical potential theory. J Comput Phys, 1985, 60: 187-207

5 Board J, Schulten K. The fast multipole algorithm. IEEE Comput Sci Eng, 2000, 2(1): 76-79

6 Liu Y J, Nishimura N. The fast multipole boundary element method for potential problems: A tutorial. Eng Anal Bound Elem, 2006, 30: $371-381$

7 Nishimura N. Fast multipole accelerated boundary integral equation methods. Appl Mech Rev, 2006, 55(4): 299-324

8 Mei C C. The applied dynamics of ocean surface waves. Singapore: World Scientific Publishing Co. Pte. Lte., 1989. 315-316

9 Abramowitz M, Stegun I A. Handbook of Mathematical Functions. New York: Dover, 1965

10 Saad Y, Schultz M. GMRES: A Generalized minimal residual algorithm for solving nonsymmetric linear systems. SIAM Sci Stat Comput, 1986, 7: 856-859

\section{Diffraction of linear waves around a vertical circular cylinder using the FMBEM}

\author{
LIN ZhiLiang* \\ State Key Lab of Ocean Engineering School of Naval Architecture Ocean and Civil Engineering, Shanghai JiaoTong University \\ Shanghai 200240, China
}

A numerical solution for the diffraction of linear waves around a vertical cylinder is presented in this paper using the fast multipole boundary element method (FMBEM). The fast multipole method (FMM) is employed to accelerate the traditional boundary element method. The accuracy and efficiency of the FMBEM for 2D Helmholtz equation is demonstrated, and it's proved that this approach is suitable for solving large-scaled problems. In addition, numerical results of water dynamics coefficients, which are related to the diffraction problems of linear waves around a cylinder, are supplied in this paper.

fast multipole boundary element method, wave diffraction, Helmholtz equation

PACS: 4 7.35.Lf, 71.15.Ap, 92.10.Hm, 92.10.Lq

doi: $10.1360 / 132010-1067$ 